\title{
Actitudes hacia el alumnado con discapacidad en educación física: validación de la EAADEF-EP a la etapa de educación primaria
}

\author{
Attitudes toward students with disabilities in physical education: \\ validation of the EAADEF-EP to the stage of primary education

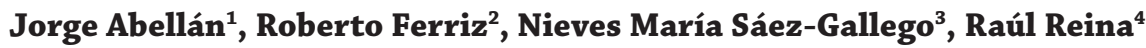 \\ 1 Facultad de Educación de Cuenca. Universidad de Castilla-La Mancha. España. \\ 2 Facultad de Ciencias Sociales y Humanas de Teruel, Universidad de Zaragoza. España. \\ 3 Facultad de Educación de Toledo. Universidad de Castilla-La Mancha. España. \\ 4 Facultad de Ciencias Sociosanitarias, Centro de Investigación del Deporte. Universidad Miguel Hernández de Elche. España. \\ CORRESPONDENCIA: \\ Roberto Ferriz \\ roberto.ferriz@unizar.es \\ Recepción: junio 2019 • Aceptación: octubre 2019 \\ CÓMO CITAR EL ARTÍCULO: \\ Abellán, J., Ferriz, R., Sáez-Gallego, N. M., \& Reina, R. (2020). \\ Actitudes hacia el alumnado con discapacidad en educación física: \\ Validación de la EAADEF-EP a la etapa de educación primaria. \\ Cultura, Ciencia y Deporte, 15(44), 235-243.
}

\section{Resumen}

La investigación que aborda la inclusión del alumnado con discapacidad en educación física (EF) se presenta como un reto educativo en la actualidad. El objetivo de este estudio fue validar la Escala de Actitud hacia el Alumnado con Discapacidad en Educación Física (EAADEF) de Íñiguez-Santiago et al. (2017) a la etapa de educación primaria (EAADEF-EP). Participaron 465 estudiantes (rango 9-13 años) que cursaban $4^{\circ}, 5^{\circ}$ y $6^{\circ}$ curso de la etapa de educación primaria obligatoria (EPO). Se confirmaron unos índices de ajuste adecuados para la adaptación de la EAADEF a EPO, mostrándose la estructura factorial invariante respecto al curso, género, el contacto previo con familiares o amigos con discapacidad, el contacto con compañeros con discapacidad en clase de EF, así como por haber participado en una actividad deportiva con personas con discapacidad. Se obtuvieron valores excelentes de consistencia interna de los ítems $(\alpha=.79)$ y del constructo $(\omega=.98)$. Las correlaciones entre los ítems de la escala, así como la correlación entre los ítems y la puntuación media de actitud, fueron positivas y significativas. El análisis de validez de criterio reveló que los chicos presentaron una actitud más positiva hacia sus iguales con discapacidad en EF.

Palabras clave: inclusión, sensibilización, validez, propiedades psicométricas.

\begin{abstract}
The research that addresses the inclusion of students with disabilities in physical education (PE) is an educational challenge at present. The aim of this study was to validate the Attitudes' Scale towards Students with Disabilities in Physical Education from Íniguez-Santiago et al. (2017) at the compulsory primary education stage (PES) (EAADEF-EP). 465 students took part in this study (range $9-13$ years old), enrolled in 4th, 5th and 6th grade of the PES. Adequate adjustment indexes were confirmed for the adaptation of the EAADEF-EP to this education stage, showing an invariant factor structure with respect to the course, gender, contact with family members or friends with disabilities, contact with a partner with a disability in PE, as well as for having participated in a sports activity with people with disabilities. Excellent values of internal consistency were obtained for the items $(\alpha=.79)$ and the construct $(\omega=.98)$. The correlations between the items of the scale as well as the correlation between the items and the average attitude score were positive and significant, with values between .43 and .84 . The analysis of criterion validity revealed that boys presented a more positive attitude towards their peers with disabilities in PE.
\end{abstract}

Key words: inclusion, sensitization, validity, psychometric properties. 


\section{Introducción}

La inclusión educativa de personas con discapacidad es un derecho reconocido, recogido en la ley educativa vigente (LOMCE, 2013), que aboga por una igualdad de derechos y oportunidades que ayude a superar cualquier discriminación y la accesibilidad universal a la educación; elementos ratificados a su vez por la Convención sobre los Derechos de las Personas con Discapacidad (ONU, 2006). En España, los últimos datos disponibles del Ministerio de Educación, Cultura y Deporte (MECD) para el curso 2016/2017, indican que el total de alumnado con discapacidad incluido en centros educativos donde se cursa la etapa de educación primaria obligatoria es de 87.835 , dato que representa el 2,9\% del total de estudiantes matriculados en esta etapa (MECD, 2018). En este contexto, las actitudes de los compañeros han sido identificadas como uno de los factores clave para afrontar este reto con garantías (Ocete at al., 2017). Y en este ámbito educativo, la asignatura de Educación Física (EF) ha sido descrita como un área propicia para que alumnado con y sin discapacidad compartan experiencias positivas (Campos, Ferreira, \& Block, 2014). Así, percibir una actitud positiva por los iguales ha sido valorada por los propios estudiantes con discapacidad como un comportamiento fundamental para sentirse incluidos (Spencer-Cavaliere \& Watkinson, 2010).

Se entiende por actitud una idea que predispone a adoptar un determinado comportamiento en situaciones sociales (Triandis, 1971). Inicialmente este concepto se definió como un constructo teórico de tres componentes: cognitivo (idea), afectivo (emoción) y conductual (predisposición a la acción). Como indican (Ocete at al., 2017) es necesario discutir esta categorización inicial de Triandis (1971) debido al desarrollo de multitud de investigaciones aplicadas a un contexto real de inclusión, ya que parece que las tres dimensiones del concepto responden más a una categorización teórica que práctica de evaluación de las actitudes hacia la discapacidad (De Boer, Timmerman, Pijl, \& Minnaert, 2012). La evaluación de las actitudes hacia la discapacidad en las clases de EF sigue siendo objeto de interés e investigación actualmente (e.g. Haegele \& Sutherland, 2015; McKay, Park, \& Block, 2018; Reina et al., 2019). Sin embargo, la cantidad de instrumentos disponibles en la literatura son escasos, especialmente en castellano, y más concretamente si se quiere evaluar las actitudes en las primeras etapas de la educación obligatoria (i.e., educación primaria) en las que parece clave obtener retroalimentación sobre la efectividad de la intervención docente o la propia inclusión en el aula de EF.
Para poder investigar acerca de las actitudes hacia el alumnado con discapacidad es necesario disponer de instrumentos de medida válidos y fiables que puedan ser administrados, al menos, en las etapas educativas que forman parte de la educación obligatoria. Sin embargo, en la actualidad son limitados los instrumentos disponibles para las primeras etapas educativas en las que: a) el alumnado puede adquirir actitudes que pueden mantener en la adultez, y b) se pueda evaluar la efectividad de intervenciones para fomentar una educación inclusiva. Además, la misma categorización de los componentes de la actitud ha sido objeto de discusión mediante la creación y validación de diferentes instrumentos. En el contexto español, para la etapa de educación secundaria obligatoria, se han propuesto instrumentos con dos (componente cognitivo y conductual: Reina et al., 2016) y una dimensión (componente conductual: Íñiguez-Santiago et al., 2017) del constructo de actitud.

En los últimos años, también han surgido nuevos instrumentos para poder ser empleados en el contexto español siguiendo dos procedimientos. Por un lado, existen adaptaciones al castellano de escalas internacionales, como el Children's Attitudes Towards Integrated Physical Education Revised (CAIPE-R; Block, 1995), dando lugar al CAIPE-SP (Cordente-Mesas et al., 2016) o la Chedoke-McMaster Attitudes Toward Children with Handicaps (CATCH; Rosenbaum, Armstrong, \& King, 1986) adaptada al contexto español por Felipe-Rello (2017). Por otro lado, se han creado otras escalas específicas en castellano, como la Attitudes Towards Inclusion of Students with Disabilities in Physical Education Scale (AISDPE) (Reina et al., 2016) orientada a evaluar las actitudes hacia la discapacidad en general; o específicos para un tipo de discapacidad como el Cuestionario para Evaluar las Actitudes hacia la Discapacidad Visual de los escolares (Cordente-Mesas et al., 2018).

Aunque algunos de estos instrumentos se encuentran validados para la población escolar de 9 a 12 años (e.g., ver Cordente-Mesas et al., 2016; 2018) no existe un consenso sobre su adecuación para ser administrados a esta población, ya que su elevado número de ítems y estructura multidimensional podrían dificultar su comprensión y solapamiento de los constructos a medir, pudiendo así comprometer su fiabilidad. En este sentido, Saloviita (2015) apunta que sería recomendable emplear instrumentos breves y unidimensionales, siempre que muestren evidencias de validez y fiabilidad.

En este contexto surge la Escala de Actitud hacia el Alumnado con Discapacidad en Educación Física (EAADEF; Íñiguez-Santiago et al., 2017), que se presenta como un instrumento breve, válido y fiable 
para evaluar las actitudes hacia la discapacidad en el alumnado de educación secundaria obligatoria. Dicho instrumento evalúa el componente conductual de las actitudes a través de la formulación de cuatro ítems para un contexto de clase de EF. Además, la EAADEF se presenta en la literatura como el único instrumento que evalúa la actitud hacia la discapacidad en EF invariante respecto a: a) el género, b) tener contacto con familiares o amigos con discapacidad, c) tener contacto con compañeros con discapacidad en EF y d) haber participado anteriormente en una actividad deportiva con personas con discapacidad. Tras su validación, la escala ha sido utilizada para evaluar las actitudes hacia la discapacidad del alumnado de secundaria (ver Abellán, Sáez-Gallego, \& Reina, 2018a) y para evaluar el efecto de intervenciones con el objetivo de mejorar dichas actitudes mediante la práctica de deporte inclusivo durante las clases de EF (ver Abellán, Sáez-Gallego, \& Reina, 2018b).

La presente investigación tuvo por objetivo testar la validez y fiabilidad de la EAADEF para la etapa de educación primaria. Concretamente, se analizó su estructura factorial, fiabilidad de los ítems y constructo, así como su validez de criterio, comprobando la invarianza en los valores de actitudes respecto al género, curso, participación en actividades físicas y/o deportivas y el contacto previo con personas con discapacidad, así como en función de haber tenido familiares y/o amigos y compañeros de clase de EF con discapacidad. Tomando como referencia el estudio de Íñiguez-Santiago et al. (2017), se hipotetizó que el instrumento ofrecería evidencias de su validez factorial, fiabilidad e invarianza. A su vez, se pretende contrastar los hallazgos de investigaciones previas en relación a diferentes variables demográficas también recogidas en el estudio, sugiriendo que las mejores actitudes hacia la discapacidad las mostrarán las mujeres (Litvack, Ritchie, \& Shore, 2011), los participantes de mayor edad y por tanto que asisten a cursos superiores (Aguado, Alcedo, \& Arias, 2008), así como los que han tenido un contacto previo junto a personas con discapacidad, especialmente si ese contacto corresponde a un familiar (Abellán et al., 2018a).

\section{Método}

\section{Participantes}

Cuatrocientos sesenta y cinco estudiantes de $4^{\circ}$ ( $N=157), 5^{\circ}(N=122)$ y $6^{\circ}(N=186)$ curso de educación primaria $\left(M_{\text {edad }}=11.15 \pm .94\right.$ años) formaron parte de este estudio. Respecto al género, participaron 233 chicas $\left(M_{\text {edad }}=11.23 \pm .91\right.$ años $)$ y $232 \operatorname{chicos}\left(M_{\mathrm{e}-}\right.$ $\mathrm{dad}=11.08 \pm .96$ años), que formaban parte de cinco centros educativos públicos de la zona central de España. Todos los participantes recibían tres sesiones de EF semanalmente, con una duración de 45 minutos por clase.

\section{Medidas}

Actitud hacia la discapacidad en educación física. Se utilizó la "Escala de Actitud hacia el Alumnado con Discapacidad en Educación Física” (EAADEF) de Íñiguez-Santiago et al. (2017). El instrumento está encabezado con la frase introductoria: "En educación física, con respecto a las personas con discapacidad...". Esta escala incluye cuatro ítems relacionados con el componente conductual de la actitud (e.g., "no propondría como capitán de mi equipo a una persona con discapacidad"). Las respuestas se recogen por medio de una escala Likert de 1 (totalmente en desacuerdo) a 5 (totalmente de acuerdo). Puntuaciones más altas en la EAADEF revelan una actitud menos inclusiva hacia la participación del alumnado con discapacidad en EF.

Variables sociodemográficas. Se analizaron cinco variables asociadas al género (chico o chica), curso $\left(4^{\circ}, 5^{\circ}\right.$ y $6^{\circ}$ de EPO), por haber participado en una actividad física o deportiva junto con personas con discapacidad (" ¿Has participado en alguna actividad deportiva con personas con discapacidad?”), y por haber tenido contacto con familiares o amigos con discapacidad (" $¿$ Tienes un familiar o amigo/a cercano con discapacidad?") y contacto con compañeros con discapacidad en clases de EF ("¿Has tenido alguna vez un compañero/a con discapacidad en tu clase de educación física?”). Para informar acerca del género y el curso, los estudiantes marcaban la opción correspondiente, y para el resto de las preguntas había que elegir entre dos opciones: "Sî" o "No".

\section{Procedimiento}

Se contactó con cinco centros educativos para solicitar su colaboración en el estudio. Todos los estudiantes obtuvieron el consentimiento paterno o materno para confirmar su participación en el estudio. Antes de administrar la escala, para garantizar que el alumnado comprendía el procedimiento a seguir para completar el instrumento se incluyó una pregunta de control: "Voy al colegio en localidad". La pregunta se respondía utilizando el rango de la EAADEF-EP y aquellos estudiantes que no respondieron con un 5 (totalmente de acuerdo) fueron excluidos del estudio debido a que podrían no haber entendido la forma de responder. Con este criterio, las respuestas de 23 participantes no fueron incluidas en los análisis posteriores. 
El instrumento fue administrado respetando las recomendaciones éticas de la American Psychological Association (2010), de modo que fue contestado en presencia de un miembro del grupo de investigación, que explicó la forma de completarlo, asegurando el anonimato de los participantes y la confidencialidad de sus respuestas, y resolviendo las dudas que surgieron durante el proceso de recogida de datos.

Siguiendo las directrices de Íñiguez-Santiago et al. (2017), se añadió al instrumento la explicación del concepto de discapacidad: "una persona con discapacidad es aquella, que debido a la alteración de una estructura o función corporal (física, sensorial o intelectual), tiene limitaciones a la hora de realizar las actividades que serían normales". Antes de que el alumnado respondiera el cuestionario, un miembro del grupo de investigación explicó el concepto de discapacidad, incluyendo ejemplos del día a día de un alumno o alumna con discapacidad en EF y resolviendo las dudas que pudieran surgir.

\section{Análisis de los datos}

Primero, se realizó un análisis factorial confirmatorio (AFC) para conocer la estructura factorial de la EAADEF-EP y se testó la invarianza de la escala respecto a las cinco variables sociodemográficas. Dado que para el AFC el coeficiente de Mardia fue alto (18.170), se utilizó el método de estimación de máxima verosimilitud junto con el procedimiento de bootstrapping. Los estimadores no se vieron afectados por la falta de normalidad por lo que fueron considerados robustos (Byrne, 2001). Con el objetivo de rechazar o aceptar los modelos factoriales testados, se utilizó un conjunto de varios índices de ajuste: $\chi^{2} / g l$, CFI (Comparative Fit Index), TLI (Tucker Lewis Index), IFI (Incremental Fit Index), RMSEA (Root Mean Square Error of Approximation) con su intervalo de confianza al 90\%, y SRMR (Standardized Root Mean Square Residual). Dado que el $\chi^{2}$ es muy sensible al tamaño muestral (Jöreskog y Sörbom, 1993), se empleó el $\chi^{2} / g l$, para el que se consideran aceptables valores inferiores a 3 (SchermellehEngel, Moosbrugger, \& Müller, 2003). Los índices incrementales (i.e., CFI, TLI e IFI) muestran un ajuste aceptable con valores por encima de .95 , mientras que los índices de error se consideran aceptables con valores iguales o menores de .06 para el RMSEA y .08 para el SRMR (Hu \& Bentler, 1999).

Segundo, para aceptar o rechazar la invarianza de los modelos factoriales en cuanto a las variables sociodemográficas, se compararon los índices de ajuste del modelo sin restricciones (Modelo 1) con respecto a cuatro modelos: modelo 2 = Modelo con pesos de me- dida invariantes; Modelo 3 = Modelo con covarianzas estructurales invariantes; Modelo 4 = Modelo con residuos de medida invariantes. Para aceptar la invarian$\mathrm{za}$, se requiere la ausencia de diferencias significativas entre el modelo sin restricciones (Modelo 1) y el modelo con pesos de medida invariantes (Modelo 2) (Byrne, Shavelson, \& Multen, 1989).

Tercero, se calcularon los estadísticos descriptivos, correlaciones bivariadas entre los ítems, así como entre éstos y la puntuación media de actitud, consistencia interna de la escala (alfa de Cronbach $=\alpha$ ) y del constructo de actitud (índice Omega $=\omega$ ). Se utilizaron los paquetes estadísticos Statistical Package for Social Sciences (versión 21.0 para Windows, IBM SPSS Inc, Chicago, IL, USA) y AMOS (versión, 20.0. para Windows, IBM, SPSS Inc, Chicago, IL, USA).

Cuarto, se analizó la validez de criterio para las cinco variables sociodemográficas utilizando una prueba estadística no paramétrica, tras comprobar que las variables no cumplían el supuesto de normalidad mediante la prueba de Kolgomorov-Smirnov. Por ello, para el análisis inferencial, se utilizó la prueba U Mann Whitney para analizar las diferencias en las tres dimensiones evaluadas de actitudes hacia la discapacidad en función del género y del contacto con personas con discapacidad (familiares, compañeros de clase, compañeros de Educación Física), así como en función de su participación o no en actividades físicas con este colectivo. Adicionalmente, se utilizó la prueba $K$ de KruskalWallis para explorar las diferencias en función del curso (cuarto, quinto y sexto de educación primaria). Por otra parte, se realizó el análisis correlacional mediante la prueba Rho Spearman, para determinar la relación existente entre las actitudes hacia la discapacidad y la edad. El cálculo del tamaño del efecto de las diferencias se realizó con la fórmula $(r=Z / \sqrt{ } N)$, donde $N$ es el número total de participantes en cada caso. Finalmente, se clasificó la magnitud de las diferencias significativas en diferencias pequeñas $(r=.10)$, moderadas $(r=.30)$ y grandes $(r=.50)$ (Morales, 2011).

\section{Resultados}

\section{Análisis factorial confirmatorio}

Se analizaron las propiedades psicométricas de la EAADEF-EP con el fin de rechazar o confirmar la estructura factorial original. En primer lugar, se obtuvieron los siguientes índices de ajuste: $\chi^{2}(2, N=465)$ $=8.19, p<.001 ; \chi^{2} / g l=4.10 ; \mathrm{CFI}=.99 ; \mathrm{TLI}=.97 ; \mathrm{IFI}$ $=.99 ;$ RMSEA $=.082($ IC $90 \%=.030-.143) ;$ SRMR $=$ .021. No obstante, los índices de modificación revela- 
Tabla 1. Análisis de Invarianza para las Cinco Variables Dependientes.

\begin{tabular}{|c|c|c|c|c|c|c|c|c|c|c|}
\hline Modelos & $x^{2}$ & gl & $x^{2} / g \mid$ & $\Delta \mathbf{x}^{2}$ & $\Delta \mathrm{gl}$ & CFI & TLI & IFI & RMSEA & SRMR \\
\hline \multicolumn{11}{|c|}{ Invarianza por curso } \\
\hline Modelo 1 & 5.72 & 3 & 1.91 & - & - & .99 & .97 & .99 & .000 & .013 \\
\hline Modelo 2 & 11.29 & 9 & 1.26 & 5.57 & 6 & .99 & .99 & .99 & .000 & .020 \\
\hline Modelo 3 & 11.56 & 11 & 1.05 & 5.84 & 8 & .99 & .99 & .99 & .075 & .023 \\
\hline Modelo 4 & 33.63 & 21 & 1.60 & 27.91 & 18 & .98 & .98 & .98 & .104 & .023 \\
\hline \multicolumn{11}{|c|}{ Invarianza por género } \\
\hline Modelo 1 & .17 & 2 & .09 & - & - & .99 & .99 & .99 & .000 & .005 \\
\hline Modelo 2 & 1.67 & 5 & .33 & 1.49 & 3 & .99 & .99 & .99 & .000 & .011 \\
\hline Modelo 3 & 2.21 & 6 & .37 & 2.04 & 4 & .99 & .99 & .99 & .000 & .014 \\
\hline Modelo 4 & 13.01 & 11 & 1.18 & 12.84 & 9 & .99 & .99 & .99 & .020 & .027 \\
\hline \multicolumn{11}{|c|}{ Invarianza por contacto con familiares o amigos con discapacidad } \\
\hline Modelo 1 & .33 & 2 & .163 & - & - & .99 & .99 & .99 & .000 & .008 \\
\hline Modelo 2 & 8.62 & 5 & 1.72 & $8.29 *$ & 3 & .99 & .99 & .99 & .040 & .034 \\
\hline Modelo 3 & 9.42 & 6 & 1.57 & 9.09 & 4 & .99 & .99 & .99 & .035 & .030 \\
\hline Modelo 4 & 23.76 & 11 & 2.16 & $23.43 * *$ & 9 & .98 & .98 & .98 & .050 & .046 \\
\hline \multicolumn{11}{|c|}{ Invarianza por contacto con compañeros con discapacidad en clase de educación física } \\
\hline Modelo 1 & .12 & 2 & .06 & - & - & .99 & .99 & .99 & .000 & .000 \\
\hline Modelo 2 & 8.62 & 5 & 1.72 & $8.50 *$ & 3 & .99 & .99 & .99 & .040 & .040 \\
\hline Modelo 3 & 9.34 & 6 & 1.56 & 9.22 & 4 & .99 & .99 & .99 & .035 & .035 \\
\hline Modelo 4 & 25.64 & 11 & 2.33 & $25.52 * *$ & 9 & .97 & .97 & .97 & .054 & .054 \\
\hline \multicolumn{11}{|c|}{ Invarianza por haber participado en actividades físicas o deportivas con personas con discapacidad } \\
\hline Modelo 1 & .38 & 2 & .19 & - & - & .99 & .99 & .99 & .000 & .006 \\
\hline Modelo 2 & 4.52 & 5 & .90 & 4.14 & 3 & .99 & .99 & .99 & .000 & .021 \\
\hline Modelo 3 & 5.41 & 6 & .90 & 5.04 & 4 & .99 & .99 & .99 & .000 & .028 \\
\hline Modelo 4 & 15.55 & 11 & 1.41 & 15.17 & 9 & .99 & .99 & .99 & .030 & .031 \\
\hline
\end{tabular}

Nota. Modelo 1 = Modelo sin restricciones; Modelo 2 = Modelo con pesos de medida invariantes; Modelo 3 = Modelo con covarianzas estructurales invariantes; Modelo 4 = Modelo con residuos de medida invariantes. ${ }^{*} p<.05 ; * * * p<.001$.

ron que las propiedades psicométricas de la escala podrían mejorar al correlacionar los errores de los ítems 1 y 3 presentándose los siguientes resultados apropiados (ver Anexo): $\chi^{2}(2, N=465)=.004, p=.950 ; \chi^{2} / g l$ $=.004 ; \mathrm{CFI}=.99 ; \mathrm{TLI}=.99 ; \mathrm{IFI}=.99 ; \mathrm{RMSEA}=.000$ (IC 90\% =.000 - .005); SRMR $=.0005$. Los pesos de regresión estandarizados fueron estadísticamente significativos $(p<.001)$ oscilando entre .59 y .80 .

\section{Análisis de invarianza}

En la Tabla 1 se presentan los índices de ajuste para los cuatro modelos comparados del análisis de invarianza multigrupo respecto al (1) curso, (2) género, (3) contacto con familiares o amigos con discapacidad, (4) contacto con compañeros con discapacidad en clase de EF, y (5) por haber participado en una actividad deportiva con personas con discapacidad. Para el análisis de invarianza por (3) contacto con familiares o amigos con discapacidad y (4) el contacto con compañeros con discapacidad en clase de EF se encontraron diferencias significativas en el estadístico $\chi^{2}$ entre el
Modelo 1 y los Modelos 2 y 4. Dado que el coeficiente $\chi^{2}$ es sensible al tamaño muestral, se tomó el criterio de Cheung y Rensvold (2002) respecto al $\triangle C F I$ para apoyar la invarianza respecto a ambas variables. En los análisis de invarianza respecto al (1) curso, (2) género y (5) haber participado en una actividad deportiva con personas con discapacidad, no se presentaron diferencias significativas en $\chi^{2}$ entre el Modelo 1 y los Modelos 2, 3 y 4.

\section{Estadísticos descriptivos, correlación entre ítems y análisis de fiabilidad}

Se obtuvo una puntuación media global para la actitud hacia el alumnado con discapacidad en EF por debajo del valor medio de la escala $(M=1.81 ; D T=$ .88) (ver Tabla 2). Se obtuvo una correlación positiva y moderada entre los cuatro ítems, mientras que la correlación entre cada ítem y la actitud global fue positiva con valores entre .76 y .84. La consistencia interna del instrumento fue de $\alpha=.79$ y la del constructo de $\omega=.98$. 
Tabla 2. Estadísticos Descriptivos y Correlaciones entre los Ítems de la EAADEF- EP y entre los ítems y la Media de la EAADEF- EP.

\begin{tabular}{lcccccccc}
\hline Items-Factor & $\mathbf{M}$ & DT & Asimetría & Curtosis & $\mathbf{1}$ & $\mathbf{2}$ & $\mathbf{3}$ & $\mathbf{4}$ \\
\hline 1. Ítem 1 & 1.69 & 1.03 & 1.37 & 1.07 & & .58 & .43 & .43 \\
2. Ítem 2 & 1.80 & 1.13 & 1.29 & .73 & .76 & .47 & .84 & .45 \\
3. Ítem 3 & 1.78 & 1.12 & 1.38 & 1.10 & .79 & .76 \\
4. Ítem 4 & 1.97 & 1.22 & .98 & -.07 & .78 & \\
5. EAADEF- EP & 1.81 & .88 & 1.09 & .78 & & \\
\hline
\end{tabular}

Nota. Todas las correlaciones fueron significativas $(p<.001)$.

Tabla 3. Estadísticos Descriptivos de la Actitud hacia la Discapacidad en función de cinco variables sociodemográficas.

\begin{tabular}{|c|c|c|c|c|c|c|}
\hline & & & n & $\begin{array}{l}\text { EAADEF-EP } \\
(\mathrm{M} \pm \mathrm{DT})\end{array}$ & Rango Promedio & $\mathbf{p}$ \\
\hline \multirow{2}{*}{\multicolumn{2}{|c|}{ Género }} & Chico & 232 & $1.69 \pm .88$ & 211.63 & \multirow{2}{*}{.000} \\
\hline & & Chica & 233 & $1.93 \pm .87$ & 254.28 & \\
\hline \multirow{3}{*}{\multicolumn{2}{|c|}{ Curso }} & Cuarto & 157 & $1.86 \pm .87$ & 244.69 & \multirow{3}{*}{.108} \\
\hline & & Quinto & 122 & $1.87 \pm .89$ & 241.72 & \\
\hline & & Sexto & 186 & $1.73 \pm .89$ & 217.41 & \\
\hline \multirow{2}{*}{\multicolumn{2}{|c|}{$\begin{array}{l}\text { Participación en AF y/o deportiva con persona } \\
\text { con discapacidad }\end{array}$}} & Sí & 215 & $1.78 \pm .86$ & 228.95 & \multirow{2}{*}{.535} \\
\hline & & No & 250 & $1.84 \pm .90$ & 236.49 & \\
\hline \multirow{4}{*}{ Contacto } & Familiares y/o amigos con & Sí & 295 & $1.81 \pm .88$ & 232.39 & \\
\hline & discapacidad & No & 170 & $1.81 \pm .89$ & 234.06 & \multirow{3}{*}{.688} \\
\hline & Compañero en EF con & Sí & 243 & $1.78 \pm .86$ & 227.87 & \\
\hline & discapacidad & No & 222 & $1.85 \pm .91$ & 238.61 & \\
\hline
\end{tabular}

Nota. $\mathrm{AF}=$ Actividad Física; $\mathrm{EF}$ = Educación Física

Análisis de validez de criterio. Actitud hacia la discapacidad en función de las variables sociodemográficas

Se comprobó la validez de criterio de la escala, mostrándose en la Tabla 3 los estadísticos descriptivos del valor obtenido en la EAADEF-EP hacia la discapacidad respecto al género, el curso, la participación en actividades físicas o deportivas con personas con discapacidad, y del contacto con personas con discapacidad (familiares y/o amigos y compañeros en las clases de EF).

La prueba $U$ de Mann Whitney reveló diferencias significativas en las actitudes hacia la discapacidad en función del género $(U=22069.5, Z=-3.520, p=.000$, $r=.14$ ), presentando las chicas una actitud más baja respecto a los chicos. Concretando las diferencias de género por curso, las chicas presentan puntuaciones significativamente más bajas que los chicos en quinto $(U=1279.0, Z=-2.786, p=.005, r=.25)$ y sexto curso $(U=3567.5, Z=-2.099, p=.036, r=.15)$. En ambos casos, los chicos son los que presentan una actitud más favorable. Para el resto de las variables sociodemográficas analizadas no se revelaron diferencias significativas entre los grupos comparados.

\section{Discusión}

El propósito de este estudio fue analizar la validez y fiabilidad de la EAADEF (Íñiguez-Santiago et al., 2017) para la etapa de educación primaria, constatando que la EAADEF-EP podría ser empleada en esta etapa educativa ( $4^{\circ}, 5^{\circ}$ y $6^{\circ}$ curso). Considerando que el estudio original de Íñiguez-Santiago et al. (2017) fue aplicado en la etapa de educación secundaria obligatoria y primer curso de la educación secundaria post-obligatoria, se dispone ahora de un instrumento válido y fiable que podría ser administrado durante la educación obligatoria y post-obligatoria en España, abarcando el rango de edad entre los 9 y 18 años. Este estudio ofrece pues la posibilidad de contar con un instrumento breve, de fácil comprensión y aplicación para indagar en el efecto de intervenciones en educación primaria en relación a las actitudes hacia el alumnado con discapacidad en las clases de EF.

El AFC con el que se testó la estructura de un factor formada por el componente conductual que se sustenta en los postulados de Triandis (1971) obtuvo índices de ajuste excelentes, aunque fue necesario correlacionar los errores de dos ítems debido a valores obtenidos por encima de lo recomendado para $\chi^{2} / g l$ el RMSEA $\left(\chi^{2} / g l<3\right.$; RMSEA $\left.\leq .06\right)$. Además de que la escala revele mejores índices de ajuste en la versión para primaria comparando con la versión original, también todos los pesos de regresión estandarizados de los cuatro ítems de la EAADEF-EP resultaron mejores con valores entre .59 y .80 , frente a valores entre .57 y .77 de la EAADEF aplicada en educación secundaria (Íñiguez-Santiago et al., 2017). 
Los resultados del análisis multigrupo indican que, al igual que sucedió con la EAADEF, la estructura de la EAADEF-EP también resultó invariante respecto a cuatro variables sociodemográficas (i.e., género, contacto con familiares o amigos con discapacidad, contacto con compañeros con discapacidad en clase de EF, y por haber participado en una actividad deportiva con personas con discapacidad). Además, en el presente estudio se aporta como novedad la invarianza de la escala por curso académico, una evidencia de validez que puede ser interesante teniendo en cuenta que la corta edad de los estudiantes podría haber sido una limitación a la hora de confirmar la validez y fiabilidad del instrumento. De hecho, uno de los puntos fuertes de la EAADEF comparada con otros instrumentos (e.g., CAIPE-SP de Cordente-Mesas et al., 2016; CAIPE-R de Ocete et al., 2017; AISDPE de Reina et al., 2016) radica en la posibilidad de comparar sus puntuaciones medias en cuanto a diferentes variables sociodemográficas que parecen clave o modular las actitudes hacia la discapacidad en un contexto de EF. Por tanto, el análisis de invarianza ofreció garantías para que futuros trabajos puedan comparar la actitud hacia el alumnado con discapacidad en EF en relación con las variables sociodemográficas analizadas.

En cuanto a los análisis de fiabilidad, se obtuvieron valores aceptables (> .79) de alfa de Cronbach e índice Omega. Estos valores son ligeramente superiores a los obtenidos en el artículo original (> .77) con una población de estudiantes de educación secundaria (Íñiguez-Santiago et al., 2017). Además, del análisis correlacional cabe destacar que se reveló una correlación moderada y positiva entre todos los ítems, lo que sugiere que no se produce un solapamiento entre ellos a la hora de aportar a la medida del constructo.

El análisis de la validez de criterio, utilizando las cinco variables sociodemográficas en relación con la actitud hacia la discapacidad, aporta también datos dignos de mención. Aunque no aparecen diferencias significativas para todas las variables analizadas, únicamente para la variable género, cabría indicar la tendencia mostrada, donde las actitudes hacia la discapacidad en EF son mejores a mayor edad (los alumnos de sexto presentan mejores actitudes que los de quinto y cuarto, aunque los de cuarto muestran mejores actitudes hacia la discapacidad que los de quinto) y cuando hay un contacto previo con personas con discapacidad, en la línea de investigaciones previas (i.e., Pérez-Tejero et al., 2012, Suriá, 2011). En cambio, los resultados en función del género merecen una atención más detallada. En el presente trabajo, los chicos presentan valores significativamente más positivos para la actitud que las chicas. Muchas de las investigaciones precedentes habían encontrado resultados opuestos, en los que las chicas presentaban mejores que los chicos (e.g., Abellán et al, 2018b; Block, 1995; Reina et al., 2011; Vignes et al., 2009). Sin embargo, otros autores han informado que el género no era relevante a la hora de predecir una determinada actitud hacia la discapacidad (Aguado, Alcedo, \& Arias, 2008; Cordente-Mesas et al., 2016; Panagiotou et al., 2008). Parece que en esta muestra los chicos podrían haber recibido una educación más "coeducativa” e inclusiva con respecto a la educación que haya podido recibir el estudiantado de etapas posteriores, hecho que pudiera haber reducido esas diferencias de género que apuntan algunos autores, o los mejores valores obtenidos habitualmente por las chicas. A la luz de estos resultados, parece relevante seguir trabajando en esta línea para determinar la posible interacción entre el género y las actitudes hacia la discapacidad en EF en la etapa de educación primaria.

Se sugiere que futuras investigaciones comprueben si el instrumento puede ser empleado en diferentes poblaciones de estudio (i.e., otras zonas del país $\mathrm{u}$ otras titularidades de los colegios de procedencia como concertados o privados), así como el efecto de programas de cambio de actitudes en EF en la etapa de educación primaria haciendo uso de la EAADEFEP. El hecho de que la EAADEF-EP permita su utilización en el rango de edad 9-18 años también puede permitir el estudio longitudinal de la evolución de las actitudes a lo largo de la etapa educativa obligatoria. Además, se sugiere que nuevos trabajos analicen la estabilidad temporal de la escala, especialmente respecto a la educación primaria considerando la corta edad de los estudiantes.

En resumen, la EAADEF-EP presenta mejores evidencias de validez y fiabilidad respecto a la versión original, no obstante, se sugiere que futuros trabajos analicen la fiabilidad de la escala respecto a su estabilidad temporal, así como su validez de criterio a la hora de predecir variables relacionadas con la actitud hacia la discapacidad en EF (e.g. novedad, duración del contacto, tipo de discapacidad con la que se tiene contacto). Especialmente interesante es su aplicación al contexto educativo real, ya que la brevedad y unidimensionalidad de la escala permite su utilización como instrumento de evaluación en la realización de unidades didáctica y otras intervenciones educativas, conectando así la investigación con la docencia real de EF en educación primaria. 


\section{Anexo}

Escala de Actitud hacia el Alumnado con Discapacidad en Educación Física para la etapa de Educación Primaria (EAADEF-EP)

\begin{tabular}{lcc}
\hline \multicolumn{1}{c}{ Ítems } & Pesos de regresión estandarizados & Varianzas residuales \\
\hline $\begin{array}{l}\text { Ítem 1. Prefiero no relacionarme con personas con } \\
\text { discapacidad. }\end{array}$ & .73 & .47 \\
$\begin{array}{l}\text { Ítem 2. Evitaría hacer un trabajo de clase con una persona con } \\
\text { discapacidad. }\end{array}$ & .80 & .37 \\
Ítem 3. Evitaría para mi equipo a una persona con & .75 & .43 \\
discapacidad. & .59 & .65 \\
Ítem 4. No propondría como capitán de mi equipo a una \\
persona con discapacidad.
\end{tabular}

\section{BIBLIOGRAFÍA}

Abellán, J., Sáez-Gallego, N. M., \& Reina, R. (2018b). Explorando el efecto del contacto y el deporte inclusivo en Educación Física en las actitudes hacia la discapacidad intelectual en los estudiantes de secundaria. RICYDE. Revista Internacional de Ciencias del Deporte, 53, 233-242. doi:10.5232/ricyde2018.05304

Abellán, J., Sáez-Gallego, N. M., \& Reina, R. (2018a). Evaluación de las actitudes hacia la discapacidad en Educación Física: Efecto diferencial del sexo, contacto previo y la percepción de habilidad y competencia. Cuadernos de Psicología del Deporte, 18(1), 133-140.

American Psychological Association (2010). Publication Manual of the American Psychological Association (6 ${ }^{\mathrm{a}}$ ed.). Washington, DC: American Psychological Association.

Ato, M., López, J. J., \& Benavente, A. (2013). Un sistema de clasificación de los diseños de investigación en psicología. Anales de Psicología, 29(3), 1038-1059. doi:10.6018/analesps.29.3.178511

Byrne, B. M. (2001). Structural equation modeling with Amos: Basic concepts, applications and programming. Mahwah, NJ: Erlbaum.

Byrne, B. M., Shavelson, R. J., \& Muthén, B. (1989). Testing for the equivalence of factor covariance and means structures: The issue of partial measurement invariance. Psychological Bulletin, 105, 456-66. doi:10.1037/0033-2909.105.3.456

Cordente, D., González-Víllora, S., Pastor-Vicedo, J. C., \& Contreras, O. (2018). Diseño y validación de un cuestionario para valorar las actitudes de los escolares hacia la discapacidad visual. Cuadernos de Psicología del Deporte, 18(1), 123-132.

Cordente-Mesas, D., González-Víllora, S., Block, M. E., \& Contreras-Jordán, O. R. (2016). Structure, validity and reliability of the Children's Attitudes towards Integrated Physical Education-Spanish version (CAIPE-SP). European Journal of Adapted Physical Activity, 9(2), 3-12. doi:10.5507/euj.2016.005

De Boer, A., Timmerman, M., Pijl, S. J., \& Minnaert, A. (2012). The psychometric evaluation of a questionnaire to measure attitudes towards inclusive education. European Journal of Psychology of Education, 27(4), 573-589. doi:10.1007/s10212-011-0096-z

Felipe, C., \& Garoz, I. (2014). Actividad físico-deportiva en programas de cambio de actitudes hacia la discapacidad en edad escolar: Una revisión de la literatura. Cultura, Ciencia y Deporte, 27(9), 199-210. doi:10.12800/ccd.v9i27.462

Felipe-Rello, C. (2017). Actitudes hacia la discapacidad: análisis comparativo del efecto de tres programas de sensibilización en educación física (Tesi doctoral inédita). Madrid, España: Universidad Autónoma de Madrid.

Flórez, M. A., León, A., \& Alcedo, M. A. (2009). Revisión y análisis de los programas de cambio de actitudes hacia personas con discapacidad. Anuario de Psicología Clínica y de la Salud, 5, 85-98.

García-Fernández, J. M., Inglés, C. J., Vicent, M., Gonzálvez, C., \& Mañas, C. (2013). Actitudes hacia la discapacidad en el ámbito educativo a través del SSCI (2000-2011). Análisis Temático y Bibliométrico. Elec- tronic Journal of Research in Educational Psychology, 11(1), 139-166. doi:10.25115/ejrep.v11i29.1561

González, J., \& Baños, L. M. (2012). Estudio sobre el cambio de actitudes hacia la discapacidad en clases de actividad física. Cuadernos de Psicología del Deporte, 12(2), 101-108. doi:10.4321/S157884232012000200011

González, J., \& Cortés, R. (2016). Actitudes y creencias hacia la discapacidad en clases de educación física. Una cuestión educativa. Psychology, Society \& Education, 8(2), 105-120. doi:10.25115/psye.v8i2.454

Haegele, J. A., \& Sutherland, S. (2015). Perspectives of students with disabilities toward physical education: a qualitative inquiry review. Quest, 67(3), 255-273. doi:10.1080/00336297.2015.1050118

Hu, L., \& Bentler, P. M. (1999). Cut-off criteria for fit indexes in covariance structure analysis: Conventional criteria versus new alternatives. Structural Equation Modelling, 6, 1-55. doi:10.1080/ 10705519909540118

Íñiguez-Santiago, M. C., Ferriz, R., Martínez-Galindo, M. C., CebriánSánchez, M. M., \& Reina, R. (2017). Análisis factorial de la escala de actitudes hacia el alumnado con discapacidad en educación física (EAADEF). Psychology, Society, \& Education, 9(3), 493-504. doi:10.25115/ psye.v9i3.652

Jöreskog, K. G., \& Sörbom, D. (1993). LISREL 8: Structural equation modeling with the SIMPLIS command language. Chicago, IL: Scientific Software.

Ley Orgánica 8/2013, de 9 de diciembre, para la mejora de la calidad educativa. Boletín Oficial del Estado, 10 de diciembre de 2013.

Lindsay, S., \& Edwards, A. (2012). A systematic review of disability awareness interventions for children and youth. Disability and Rehabilitation, 25, 1-24.

Litvack, M. S., Ritchie, K. C., \& Shore, B. M. (2011). High and averageachieving students' perceptions of disabilities and of students with disabilities in inclusive classrooms. Exceptional Children, 77(4), 474487. doi:10.1177/001440291107700406

Martínez, M. A. \& Bilbao, M. C. (2011). Los docentes de la universidad de Burgos y su actitud hacia las personas con discapacidad. Revista Española sobre la Discapacidad Intelectual Siglo Cero, 42(4), 50-78.

McKay, C., Park, J. Y., \& Block, M. (2018). Exploring the variables associated with student attitudes toward inclusion in physical education after taking part in the Paralympic School Day programme. International Journal of Inclusive Education. doi:10.1080/13603116.2018.1550117

Ministerio de Educación, Cultura y Deportes (MECD) (2018). Estadística de las enseñanzas no universitarias. Alumnado con necesidad específica de apoyo educativo. Recuperado el 11/02/2019 de http://www.educacionyfp.gob.es/servicios-al-ciudadano-mecd/en/estadisticas/educacion/ no-universitaria/alumnado/necesidades-apoyo/2016-17.html

Morales, P. (2011). Tamaño necesario de la muestra ¿Cuántos sujetos necesitamos? Madrid: Universidad Pontificia Comillas. 
Nowicki, E. (2006). A cross-sectional multivariate analysis of children's attitudes towards disabilities. Journal of Intellectual Disability Research, 50(5), 335-348. doi:10.1111/j.1365-2788.2005.00781.x

Ocete, C., Pérez-Tejero, J., Franco, E., \& Coterón, J. (2017). Validación de la versión española del cuestionario "Actitudes de los alumnos hacia la integración en educación física (CAIPE-R)”. Psychology, Society, \& Education, 9(3), 447-458. doi:10.25115/psye.v9i3.1025

Organización de la Naciones Unidas (2006). Convención sobre los derechos de las personas con discapacidad. En: https://www.un.org/spanish/disabilities/default.asp?id=497

Pérez-Tejero, J., Ocete-Calvo, C., Ortega-Vila, G., \& Coterón, J. (2012). Diseño y aplicación de un programa de intervención de práctica deportiva inclusiva y su efecto sobre la actitud hacia la discapacidad: El Campus Inclusivo de Baloncesto. Revista Internacional de Ciencias del Deporte, 23, 258-271. doi:10.5232/ricyde2012.02905

Reina, R., Hutzler, Y., Íniguez-Santiago, M. C., \& Moreno-Murcia, J. A. (2016). Attitudes towards inclusion of students with disabilities in physical education questionnaire (AISDPE): A two component scale in Spanish. European Journal of Human Movement, 36, 75-87.

Reina, R., Hutzler, Y., Íñiguez-Santiago, M. C., \& Moreno-Murcia, J.A. (2019). Student attitudes toward inclusion in physical education: The impact of ability beliefs, gender, and previous experiences. Adapted Physical Activity Quarterly, 36(1), 132-149. doi:10.1123/ apaq. 2017-0146
Reina, R., López, V., Jiménez, M., García-Calvo, T., \& Hutzler, Y. (2011). Effects of awareness interventions on children's attitudes toward peers with a visual impairment. International Journal of Rehabilitation Research, 34(3), 235-242. doi:10.1097/MRR.0b013e3283487f49

Saloviita, T. (2015). Measuring pre-service teachers' attitudes towards inclusive education: Psychometric properties of the TAIS scale. Teaching and Teacher Education, 52, 66-72. doi:10.1016/j.tate.2015.09.003

Santana, P., \& Garoz, I. (2013). Actitudes hacia la discapacidad e intervención docente desde el deporte adaptado. Revista Internacional de Medicina y Ciencias de la Actividad Física y el Deporte, 13(49), 1-17.

Schermelleh-Engel, K., Moosbrugger, H., \& Müller, H. (2003). Evaluating the fit of structural equation models: tests of significance and descriptive goodness-of-fit measures. Methods of Psychological Research Online, 8(2), 23-74.

Spencer-Cavaliere, N., \& Watkinson, E. J. (2010). Inclusion understood from the perspectives of children with disability. Adapted Physical Activity Quarterly, 27, 275-293. doi:10.1123/apaq.27.4.275

Suriá, R. (2011). Comparative analysis of student's attitudes toward their classmates with disabilities. Electronic Journal of Research in Educational Psychology, 9, 197-216.

Triandis, H. C. (1971). Attitude and attitude change. New York, NY: John Wiley \& Sons.

Verdugo, M. A., Arias, B., \& Jenaro, C. (1994). Actitudes hacia las personas con minusvalía. Madrid: Imserso. 